\title{
Una estructura en tierra cruda en el sitio de Japoto, Ecuador. Los aportes de la arqueometría
}

Une structure en terre crue sur le site de Japoto, Équateur. Les apports de l'archéométrie

A raw-earth structure on the Japoto site. The contribution of archaeometry

\section{Véronique Wright}

\section{(2) OpenEdition}

Journals

\section{Edición electrónica}

URL: http://journals.openedition.org/bifea/1777

DOI: 10.4000/bifea. 1777

ISSN: 2076-5827

\section{Editor}

Institut Français d'Études Andines

\section{Edición impresa}

Fecha de publicación: 1 diciembre 2010

Paginación: 563-575

ISSN: 0303-7495

\section{Referencia electrónica}

Véronique Wright, « Una estructura en tierra cruda en el sitio de Japoto, Ecuador. Los aportes de la arqueometría », Bulletin de l'Institut français d'études andines [En línea], 39 (3) | 2010, Publicado el 01 junio 2011, consultado el 08 diciembre 2020. URL : http://journals.openedition.org/bifea/1777 ; DOI : https://doi.org/10.4000/bifea.1777

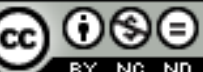

Les contenus du Bulletin de l'Institut français d'études andines sont mis à disposition selon les termes de la licence Creative Commons Attribution - Pas d'Utilisation Commerciale - Pas de Modification 4.0 International. 


\title{
Una estructura en tierra cruda en el sitio de Japoto, Ecuador. Los aportes de la arqueometría
}

\author{
Véronique Wright*
}

\begin{abstract}
Resumen
El hallazgo arqueológico de una estructura arquitectónica en tierra cruda en el sitio manteño de Japoto, llevó a desarrollar un estudio arqueométrico. A partir de unos análisis físico químicos realizados sobre un muestreo coherente, el objetivo era caracterizar los materiales de construcción empleados y luego intentar obtener indicios sobre su procedencia geológica. Por otro lado, se quiso entender por qué varias superficies arquitectónicas presentaban rasgos de rubefacción. Con los resultados obtenidos, se pudo al final desarrollar problemáticas de conservación. Las conclusiones sacadas aportaron datos inéditos y confirmaron el interés de la arqueometría en el marco de este tipo de investigaciones.
\end{abstract}

Palabras claves: manteña, Japoto, tierra cruda, arqueometría, conservación

\section{Une structure en terre crue sur le site de Japoto, Équateur. Les apports de l'archéométrie}

\section{Résumé}

La découverte d'une structure architecturale en terre crue sur le site manteño de Japoto a conduit à envisager une étude archéométrique. À partir d'analyses physico-chimiques réalisées sur un échantillonnage représentatif, il s'agissait de caractériser les matériaux de construction employés et d'obtenir des indices sur leur provenance géologique. Par ailleurs, il convient de comprendre pourquoi

* Doctora en Arqueología Precolombina, especialidad: Física aplicada en arqueología. Universidad Panthéon Sorbonne - Paris 1. Maison de l'Archéologie et de l'Ethnologie de Nanterre, UMR CNRS 8096, 21, allée de l'Université, F-92023, Nanterre Cedex. Centre de Recherche et de Restauration des Musées de France, Palais du Louvre, Porte des Lions, 14 Quai François Mitterrand, 75001 París. E-mail: vero_wright@hotmail.com 
plusieurs zones de la structure présentaient des traces de chauffage. Ces résultats permirent finalement de développer des problématiques de conservation. Les conclusions dégagées ont livré des données inédites et, ont confirmé l'intérêt de l'archéométrie dans le cadre de ce type de projets de recherche.

Mots clés : manteña, Japoto, terre crue, archéométrie, conservation

\title{
A raw-earth structure on the Japoto site. The contribution of archaeometry
}

\begin{abstract}
The discovery of an earthen architectural structure at the Manteño site of Japoto led us to develop an archaeometric study. From the physico-chemical analyses carried out on a representative sample, we characterized the building materials that were used and recovered clues on their geologic origin. We wished also to understand why several areas of the structure presented signs of heating. With these results, we were able to start developing some preservation problematics. The conclusions reached were consistent with unpublished data and confirm the value of archaeometry within the framework of this type of archaeological research project.
\end{abstract}

Key words: Manteño, Japoto, earthen structure, archaeometry, preservation

\section{INTRODUCCIÓN}

A pesar de su eficacia la herramienta arqueométrica permanece aún poco utilizada en los proyectos arqueológicos ecuatorianos. Sin embargo, los análisis fisico químicos realizados sobre muestras obtenidas en el sitio de Japoto, en la provincia de Manabí, permiten ayudar a la comprensión de varios problemas arqueológicos y confirmar el interés del uso de este tipo de estudio. Así, los resultados obtenidos después de un año de investigaciones en el C2RMF demuestran que esta técnica de trabajo complementa el estudio arqueológico, sea sobre arquitecturas, sea sobre conjuntos funerarios.

\section{LOS ESTUDIOS ARQUEOMÉTRICOS ANTERIORES}

Los estudios arqueométricos realizados sobre artefactos manteños son muy escasos, e inexistentes en el caso de vestigios construidos en tierra cruda. En este sentido, la estructura descubierta dentro del montículo J8 de Japoto autoriza un estudio inédito.

1 Centre de Recherche et de Restauration de Musées de France - Palais du Louvre, Porte des Lions, 75001 Paris. 
Sobre el sitio de Japoto, algunos análisis fueron realizados por el Centro de Investigación en Física Aplicada a la Arqueología de Burdeos en Francia2. Estos estudios fueron dedicados, en primer lugar, a las «empanadillas» descubiertas en Atacames y Japoto, descritas como «objetos de tierra arcillosa ligeramente cocida» cuya función era desconocida (Guinea, 2006: 322). Cuatro empanadillas de Japoto fueron caracterizadass: la primera estaba constituida de un limo cuarzoso con poca arcilla y elementos de calcio, potasio, sodio, magnesio y hierro. La segunda muestra era también un limo cuarzoso pero más fino y calcítico con los mismos minerales que la anterior. La tercera empanadilla estaba compuesta de un material calcítico y la cuarta de un material calcítico muy duro con inclusiones de cuarzo, feldespatos y halita (Guinea, 2006: 329). Estos resultados, junto a las observaciones y datos arqueológicos, han llevado los arqueólogos a considerar las empanadillas como tierras comestibles. Su uso principal estaría relacionado con la absorción de las fitotoxinas (Guinea, 2006: 332).

En un segundo momento, se analizaron dos muestras de suelos obtenidas de las tolas J3 y J7 del sitio de Japoto4. Los resultados indican, a pesar de pequeñas diferencias, una composición similar: un limo sin calcáreo (Platel, 2006).

Estos primeros resultados revelan las ventajas del estudio de laboratorio. En el caso de Japoto han participado en el entendimiento de la función de un artefacto muy particular como son las empanadillas, y han permitido obtener datos de base en cuanto a la composición de los suelos y así desarrollar problemáticas geológicas. Considerando el interés de estos datos inéditos, hemos desarrollado un nuevo estudio arqueométrico sobre las tolas J8 y J7 según varias problemáticas.

\section{PROBLEMÁTICAS DE LA INVESTIGACIÓN}

\section{1. La Estructura 1 del montículo J8}

Durante la temporada 2007, una estructura construida en tierra ha sido descubierta en la tola J8 y excavada en la temporada 2008 (fig. 1). Esta estructura constituye un caso excepcional dentro de la arquitectura manteña recuperada y su descripción precisa puede consultarse en la contribución de Mercedes Guinea en este mismo volumen.

Llamó especialmente la atención que sobre los adobes del murete escalonado, los taludes de refuerzo de la plataforma y el piso, fuera aplicado un enlucido

2 Los análisis han sido realizados por Nicole Platel: CRP2A - UMR CNRS 5060, Université Michel de Montaigne - Bordeaux 3.

3 Microscopía óptica, Microscopía electrónica de barrido acoplado con un sistema de análisis por dispersión de rayos X (MEB-EDS), Difracción de rayos X (DRX).

4 Análisis realizados por Nicole Platel (CRP2A), con observaciones a la lupa binocular y en microscopía de polarización, análisis elementales en MEB-EDS y análisis estructurales en DRX. 


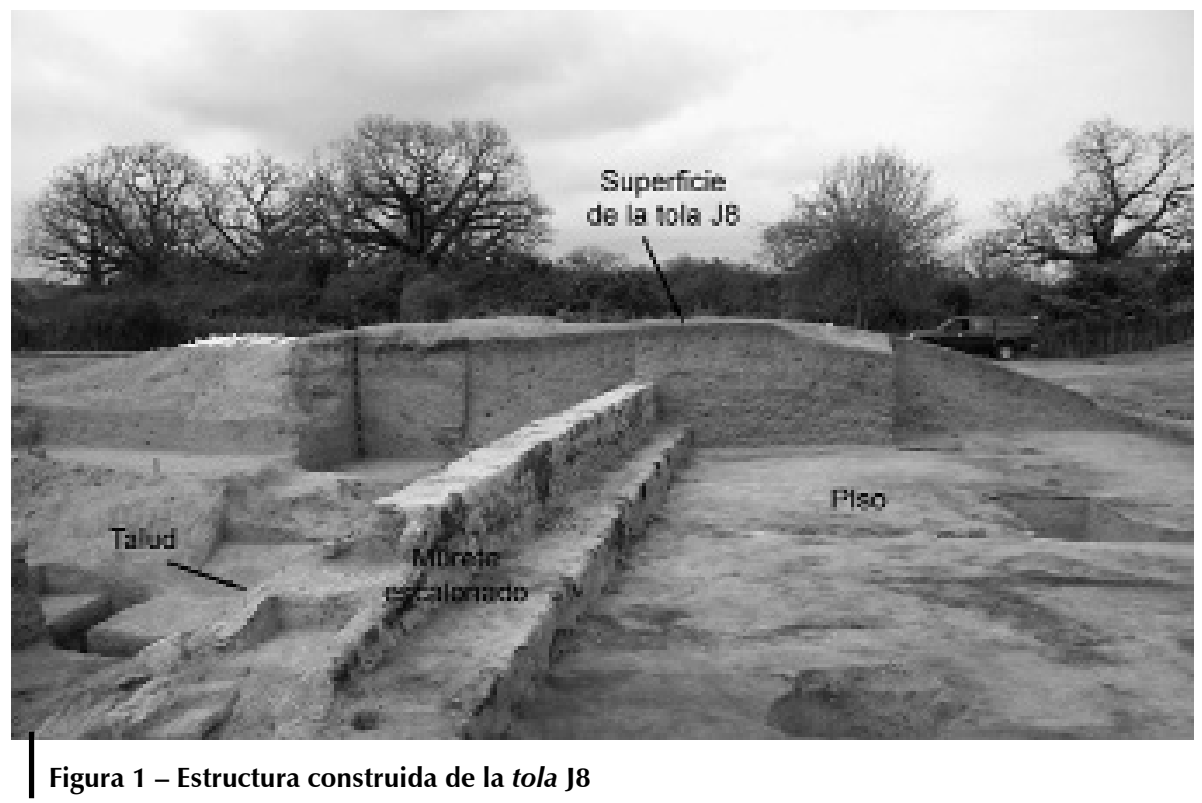

Fotografía con una vista hacia el Sur de la estructura construida descubierta en la tola J8. Permite localizar el murete escalonado, el talud al Este y el piso al Oeste (Wright V., 2008)

grueso de varios centímetros de espesor ( 2 a $9 \mathrm{~cm}$ según las zonas) que presentaba rasgos de calefacción, es decir zonas con huellas de rubefacción y de combustión. El objetivo de trabajo sobre este edificio fue doble: arqueológico y de conservación. Primero, se trataba de participar en el entendimiento de las técnicas de construcción analizando los adobes utilizados para el murete escalonado y el enlucido grueso aplicado sobre toda la estructura. Se quiso entender también el fenómeno de calefacción materializado sobre toda la estructura, estudiando muestras con huellas de rubefacción. En segundo lugar había que desarrollar propuestas de conservación. En efecto, con los resultados de composición de los materiales empleados para la construcción la idea era de proponer métodos de conservación y tratamientos químicos adaptados a las características concretas de la E1.

\section{2. El conjunto funerario de la tola J7}

Las excavaciones realizadas sobre la tola $\mathrm{J} 7$ permitieron descubrir una concentración importante de restos humanos organizados en depósitos primarios (tumbas) o secundarios (paquetes). La particularidad de esta tola era la cantidad y la variabilidad de los depósitos y la presencia exclusiva de estructuras funerarias. Considerando esta reagrupación de restos humanos parecía interesante verificar si las propiedades geomorfológicas del suelo estaban relacionadas con la preservación de los cuerpos o con su descomposición, argumentando la elección de esta tola para implantar este conjunto funerario (Delabarde, 2006: 319). En 
este sentido, se ha realizado medidas de $\mathrm{pH}$ del suelo de la tola $\mathrm{J7}$, y comparado los datos con las medidas de $\mathrm{pH}$ realizadas sobre las tolas J8 y J10 para poner en evidencia una eventual particularidad.

Luego, fue estudiada la estratigrafía de ocupación de la tola. En dos de los sondeos (PX4 y PY2) se ha realizado un muestrario de todas las capas de ocupación identificadas, de la superficie de la tola al suelo geológico, para obtener datos de su composición e intentar entender más precisamente unos depósitos grises incluidos en varias capas de esta estratigrafía.

\section{3. Conclusión}

Para desarrollar la investigación, se sacaron muestras de las tolas J8 y J75, durante la temporada de excavación 20086. El estudio arqueométrico se ha desarrollado en el C2RMF según un protocolo analítico completo que permitió obtener resultados representativos.

\section{METODOLOGÍA}

\section{1. Los métodos de observación}

Antes de cualquier análisis, cada muestra fue examinada a dos niveles de observación.

La lupa binocular (LB) permite describir la fisonomía general de las muestras según una ampliación entre 7,5 y 50 veces. Es una información de base que merece ser completada por otros métodos.

La microscopía óptica (MO) permite realizar observaciones con una ampliación x50, x100, x200 o x500, sobre cortes transversales. Para observar su sección y su estratigrafía las muestras son incluidas en resina poliéster, cortadas y pulidas.

\section{2. Los análisis elementales}

La microscopía electrónica de barrido acoplada a un sistema de análisis a dispersión de energía (MEB-EDS) ha sido aplicada sobre los cortes estratigráficos después de las observaciones en LB y MO. El MEB es una técnica de imagen que permite ampliaciones de hasta 300000 veces. Para una mejor calidad de imagen, los materiales no conductores han sido cubiertos de una fina capa de carbón o de oro según las muestras. Para el estudio, dos tipos de imágenes han sido utilizados: en

5 Se recogieron 22 muestras de la tola J8 y 17 de la tola J7.

6 La excavación duró del 12 de junio al 12 de agosto de 2008, bajo la dirección de Jean-François Bouchard (DR2 CNRS, Université Panthéon Sorbonne - Paris 1, MAE Nanterre - Archéologie des Amériques - UMR CNRS 8096) y de Mercedes Guinea (Universidad Complutense de Madrid). 
electrones secundarios, dando informaciones sobre la superficie y la topografía de la muestra; y en electrones retrodifundidos que dan una imagen cuyo contraste depende del número atómico. El sistema de análisis acoplado al MEB, indica la composición de la muestra. Es elemental porque permite identificar los elementos químicos (átomos) componiendo el material sin identificar las moléculas de las cuales forman parte. Este análisis se traduce en un espectro, representando el número de rayos $X$ detectados en función de su energía (Eberhart, 1997). Su lectura permite identificar los elementos químicos presentes en la zona de análisis nunca superior a algunos $\mu \mathrm{m}$. Esta respuesta puede también ser exportada en forma de imágenes $X$, ilustrando la repartición espacial de cada elemento químico encontrado. En el C2RMF, han sido utilizados dos aparatos MEB-EDS: un Jeol JSM-840 y un Philips XL 30CP a presión parcial, trabajando con una tensión de aceleración de 10 a $20 \mathrm{kV}$.

Esta etapa de la metodología es primordial. Permite complementar las primeras observaciones, y ofrece los primeros datos de composición en función de los cuales se orienta el resto del protocolo con una técnica de análisis estructural complementaria adaptada.

\section{3. Los análisis estructurales}

La difracción de rayos $X(\mathrm{DRX})$ es una técnica cualitativa y a veces cuantitativa empleada para caracterizar los productos minerales. Permite identificar las fases cristalinas de una muestra, sus proporciones y su grado de cristalización. Ha sido aplicada sobre muestras molidas en polvo. Para el estudio, se ha utilizado un difractómetro D5000 Brüker, en configuración $\theta-2 \theta$. Ha sido muy útil para identificar las clases de arcillas encontradas.

La espectrometría infrarroja transformada de Fourrier (IRTF) es una técnica de espectrometría vibracional cualitativa y a veces cuantitativa de las estructuras moleculares. Permite caracterizar las funciones químicas de los productos orgánicos, inorgánicos, cristalizados o amorfos. Para nuestra investigación, se ha trabajado sobre pastillas de $\mathrm{KBr}$ con un espectrómetro infrarrojo Perkin Elmer Spectrun 2000, en el medio infrarrojo (2,5 a $25 \mu \mathrm{m})$ en transmisión. Este método tiene un rol preponderante, pues es aplicable a los materiales minerales pero también orgánicos.

La microespectrometría Raman $(\mu \mathrm{R})$ es una técnica de análisis estructural óptica de espectrometría vibracional utilizando la simetría de las moléculas. Permite caracterizar los materiales orgánicos e inorgánicos, cristalinos y amorfos. Además, no necesita una preparación particular de la muestra. En laboratorio, se ha trabajado con un microespectrómetro Raman Jobin-Yvon Infinity, equipado de dos láseres visibles (633 y $532 \mathrm{~nm}$ ). La resolución espacial es $3 \mathrm{~nm}$ y espectral $2 \mathrm{~nm}$. Se utilizó principalmente el láser verde $(532 \mathrm{~nm})$ con respecto a nuestro material. En el protocolo, ha sido la última técnica de análisis estructural empleada y ha sido útil sobre todo para complementar las experimentaciones anteriores. En efecto, es 
un método no destructivo y el diámetro de la zona de análisis es muy restringido (2 à $10 \mu \mathrm{m}$ según el objetivo) permitiendo trabajar sobre superficies muy pequeñas o lagunosas. Además, es muy eficaz para diferenciar los componentes de tipo carbón, al contrario de las otras técnicas.

\section{4. Los análisis orgánicos}

Las pruebas microquímicas permiten poner en evidencia la presencia de materias orgánicas tales como las proteínas y el almidón. Consisten en poner una gota de reactivo sobre la superficie de la muestra para observar una coloración característica. Para identificar y diferenciar las proteínas, se utilizan tres reactivos del mismo colorante: el Negro Amida (Naftol Azul Negro) pero con pH distintos. Una reacción positiva se traduce por una coloración azul cuya intensidad varía en función de la concentración de proteínas. Los tres reactivos permiten diferenciar tres componentes: yema de huevo, proteínas en general y colas de tipo gelatina. La prueba para el almidón se realiza con un reactivo llamado Lugol (solución acuosa de Yodo $\left(\mathrm{I}_{2}\right)$ y de Potasio Yoduro $(\mathrm{KI})$ ). Una reacción positiva se traduce en una coloración morada a marrón de las partículas de almidón.

\section{5. Las medidas de pH}

Para realizar estas medidas hemos mezclado $2 \mathrm{~g}$ de muestra reducida en polvo con $20 \mathrm{~mL}$ de agua pura. Esta mezcla fue agitada 30 minutos con un electro-imán. Después de un tiempo de reposo de una hora, el pH es medido con una sonda a $\mathrm{pH}$, a una temperatura de 20 a $25{ }^{\circ} \mathrm{C}$.

\section{6. Conclusión}

El protocolo desarrollado requiere varias técnicas: elemental (MEB-EDS) y estructurales (DRX, IRTF y $\mu R$ ). Cada una entrega una serie de resultados, pero es la combinación de todas y el cruzamiento de los datos lo que nos permite caracterizar de manera precisa y segura la composición de una muestra. Además, permite trabajar sobre los productos minerales pero también sobre los orgánicos. Este protocolo analítico permitió, de esa forma, estudiar la totalidad del material seleccionado en las dos tolas.

\section{LOS RESULTADOS}

\section{1. La estructura E1 del montículo J8}

En primer lugar hemos estudiado los adobes utilizados para la construcción del murete escalonado (fig. 2). Los análisis, en particular en espectrometría 


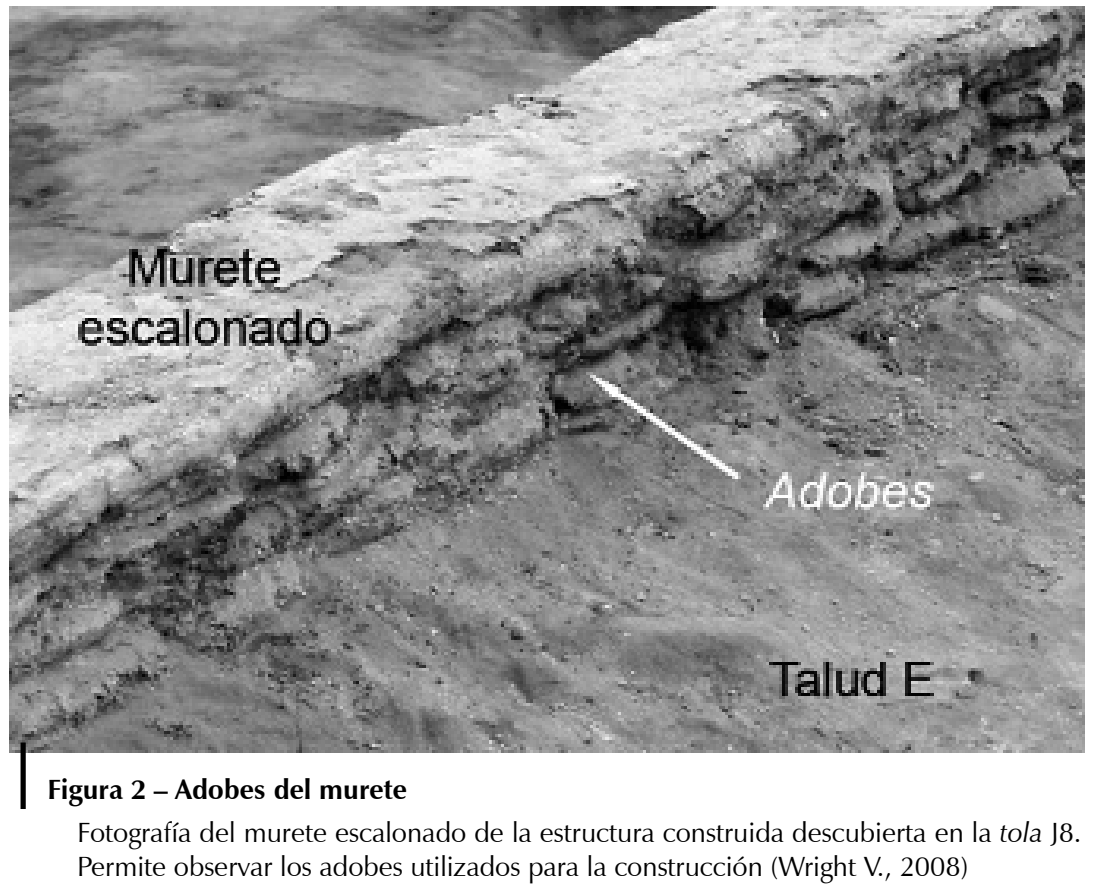

infrarroja, han permitido concluir que están compuestos esencialmente de cuarzo, feldespatos, y una arcilla. La arcilla fue identificada como ilita $\left(\left(\mathrm{K}_{1} \mathrm{H}_{3} \mathrm{O}\right)\right.$ $\left.(\mathrm{Al}, \mathrm{Mg}, \mathrm{Fe})_{2} \mathrm{O}_{10}\left[(\mathrm{OH})_{2},\left(\mathrm{H}_{2} \mathrm{O}\right)\right]\right)$, un mineral arcilloso potásico generalmente grisblanco a verdoso.

A continuación hemos analizado el enlucido grueso con huellas de calefacción, aplicado sobre el murete escalonado, los taludes y el piso. Las observaciones en sección permitieron notar en cada muestra una superposición de 3 capas sucesivas de granulometría y color diferentes (fig. 3).

La capa 1 presenta una granulometría fina y homogénea. Está constituida por microcristales rosados y rojos responsables de su coloración anaranjada, y por algunos microcristales amarillos, blancos y negros. Por otra parte, se pueden observar inclusiones de cristales en forma de láminas blancas. La capa 2 presenta un color más oscuro, casi marrón, provocada por la presencia de microcristales y de pequeños cristales gris, negros, rojos y blancos. Su granulometría es así más heterogénea que la capa precedente. También, se pueden observar grietas y varios huecos internos. Finalmente, la capa 3 presenta una granulometría fina y homogénea y es compacta con poca porosidad. Está constituida mayormente por microcristales, pequeños cristales negros y blancos y por algunas inclusiones amarillas y anaranjadas.

Aunque estas capas presentan una granulometría y una textura diferentes, las experimentaciones de caracterización nos indican una composición química muy similar. Cada capa está básicamente compuesta de cuarzo, de feldespatos, y de 


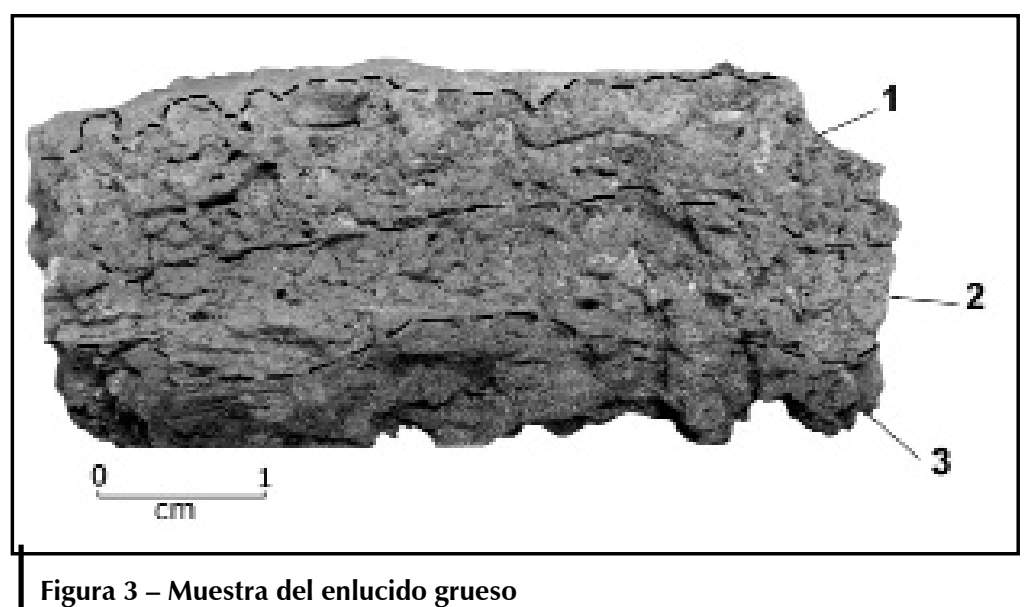

Figura 3 - Muestra del enlucido grueso

Fotografía de una muestra del enlucido grueso sacada sobre el murete escalonado. Nos permite observar la superposición de 3 capas distintas con un color y una granulometría diferentes (Wright V., C2RMF, 2008)

arcilla (ilita) o sea la misma composición que los adobes del murete escalonado. Las diferencias de color son debidas a una concentración variable de los elementos cromógenos: la hematita $\left(\mathrm{Fe}_{2} \mathrm{O}_{3}\right)$ un óxido de hierro que provoca el color anaranjado de la primera capa, la goetita $(\mathrm{FeO}(\mathrm{OH}))$ un óxido de hierro que confiere a la segunda capa su coloración más ocre, y por fin el carbón que induce el color más gris, casi negro, de la tercera capa.

Ahora bien, esta alternancia de color y de textura puede explicarse como la consecuencia de una acción de calefacción. En efecto, la combustión de esta estructura en tierra cruda ha modificado su textura y granulometría, gradualmente, en función de la difusión del calor. Así, bajo la acción del calor en superficie, la goetita de la tierra cruda se transformó en hematita7 dando este color más rojo. Por otro lado, más adentro del enlucido, la combustión de los materiales orgánicos de la tierra cruda explican la concentración más importante en carbón y el color negro.

Sobre este enlucido grueso se ha notado, en particular sobre las muestras sacadas en el murete, la presencia de una capa de color crema aplicada en superficie del enlucido grueso. Es relativamente fina (de algunos micrómetros a $200 \mu \mathrm{m}$ ) y presenta una granulometría muy fina y homogénea (del orden del $\mu \mathrm{m}$ ) que le confiere una porosidad muy débil. Está compuesta mayormente de microcristales blancos y negros y de algunos rojos y amarillos (fig. 4) y presenta una coloración verde bajo luz ultravioleta.

Los análisis indican una composición diferente de la del enlucido grueso o de los adobes. En efecto, esta capa es compuesta esencialmente de

7 Cuando se calienta alrededor de $250^{\circ} \mathrm{C}$, la goetita se transforma en hematita por deshidratación (Pomiès, 1997). 


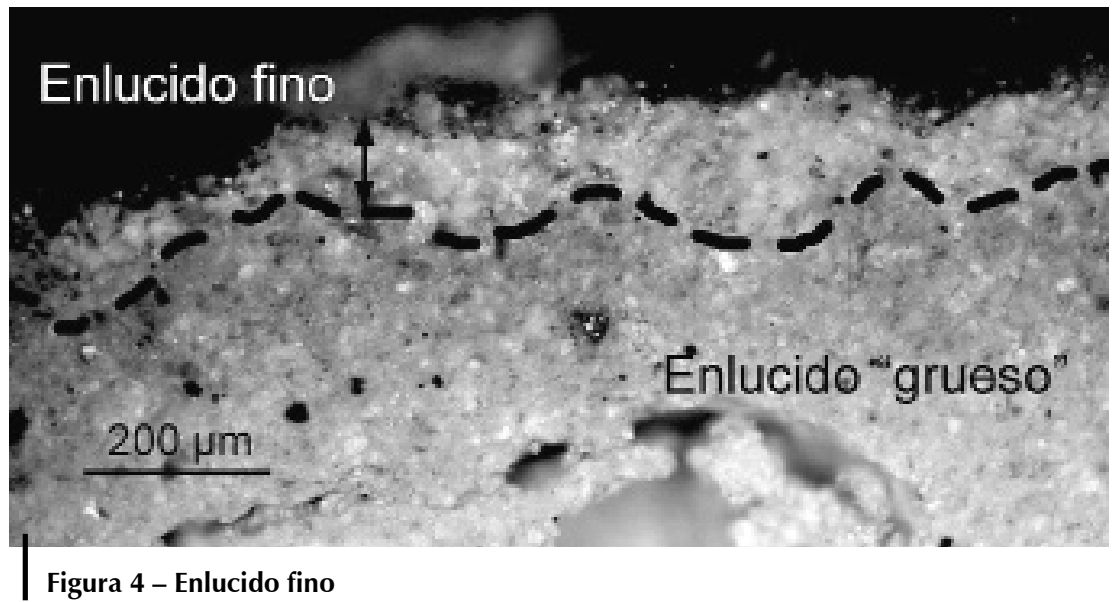

Fotografía en microscopía en sección, x100, de la capa de color crema aplicada sobre el enlucido grueso (Wright V., C2RMF, 2008)

cuarzo, feldespatos pero también de dos arcillas, la ilita y la montmorillonita $\left((\mathrm{Na}, \mathrm{Ca})_{0^{\prime} 3}(\mathrm{Al}, \mathrm{Mg})_{2} \mathrm{Si}_{4} \mathrm{O}_{10}(\mathrm{OH})_{2} \cdot \mathrm{nH}_{2} \mathrm{O}\right)$, y de carbonato de calcio $\left(\mathrm{CaCO}_{3}\right)$ que le confieren su color más blanco.

Además, las pruebas microquímicas permitieron poner en evidencia sobre varias muestras, la presencia de proteínas, traduciéndose por una coloración positiva azul. Este resultado nos permitió concluir que muy posiblemente se añadió un producto orgánico a los componentes minerales, teniendo probablemente un rol técnico de aglutinante o de cola.

Considerando todos estos datos, y el hecho que esta capa no presenta huellas de combustión, se emitió la hipótesis que este enlucido de superficie tenía un rol decorativo y ha sido aplicado como capa pictórica sobre la estructura. Sin embargo, esta propuesta queda hasta ahora una conjetura ya que los resultados obtenidos tienen que ser confirmados por análisis complementarios.

Finalmente, gracias al estudio analítico hemos podido identificar (en MEB-EDS) marcadores geológicos. Estos marcadores corresponden a inclusiones metálicas (del tipo cobre/estaño) y a monazites ((Ce,La,Nd,Th)PO4) (Wright, 2008: 117). Estas inclusiones fueron detectadas de manera escasa. Así estos datos necesitan ser profundizados pero ya nos permiten disponer de elementos de base para desarrollar hipótesis sobre la procedencia geológica.

\section{2. La tola J7}

En relación a la tola $\mathrm{J} 7$, empezamos nuestro estudio de laboratorio con medidas de $\mathrm{pH}$ sobre 14 muestras. Los resultados indican un $\mathrm{pH}$ básico entre 8,25 y 9,10. A continuación, han sido realizadas medidas de $\mathrm{pH}$ sobre muestras sacadas de las tolas $\mathrm{J} 8$ y J10. Los pH de las tolas J8 y J10 entran también en el intervalo de pH de la tola J7, pues no se pudo poner en evidencia diferencia alguna. Entonces, es 
posible proponer que las propiedades básicas del suelo de la tola $\mathbf{J} 7$ no han sido un criterio de elección para reagrupar las sepulturas en esta zona.

Prosigue el análisis de composición de las diversas capas de la estratigrafía de ocupación de la tola J7. Se ha trabajado sobre dos sondeos (PX4 y PY2), en los cuales se observó la superposición de 7 capas de sedimento (sobre unos 120 cm de altura), de color y de textura diferentes.

Los análisis indican que todas estas capas presentan una composición de base muy similar: están todas compuestas de cuarzo, feldespatos y dos arcillas (ilita y una arcilla de tipo esmectita8). Sin embargo, en los depósitos grises incluidos en estas capas se encontró una concentración en fosfatos de calcio (fig. 5). Esta concentración se verifica especialmente en la última capa de la fosa PX4 (muestras E7 y E8). Estos fosfatos de calcio corresponden muy probablemente a hidroxilapatita, una forma de apatita de calcio de formula química $\mathrm{Ca}_{5}\left(\mathrm{PO}_{4}\right)_{3}(\mathrm{OH})$. Este elemento se encuentra bajo forma mineral en la naturaleza, pero también es producido por los huesos. Estos fosfatos de calcio están ausentes en las otras capas de la tola, lo que demuestra que no se encuentran de manera natural en su suelo. Esto último lo confirman los análisis de Nicole Platel (Platel, 2006). Así, se puede proponer que esta hidroxilapatita proviene de huesos, y que algún polvo de hueso ha sido depositado en los pisos de ocupación.

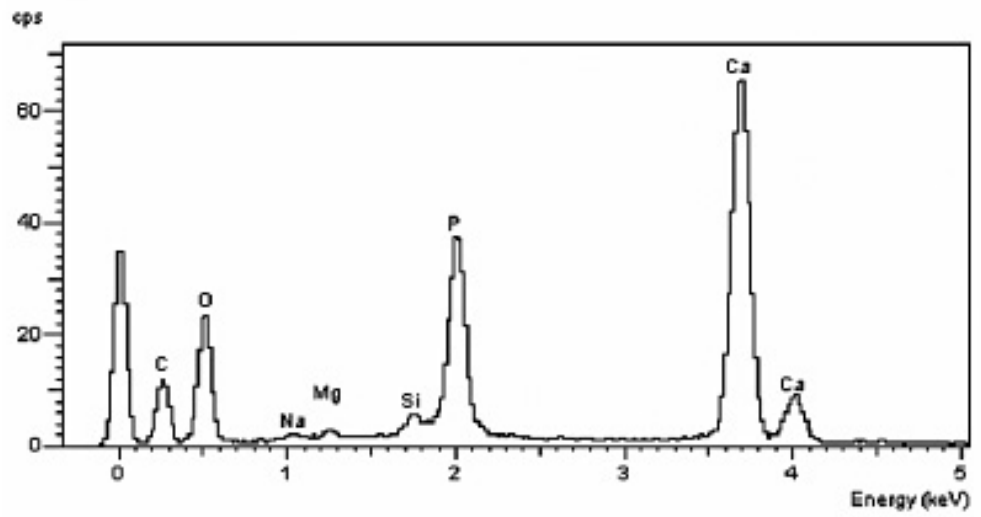

Figura 5 - Espectro EDS de fosfato de calcio

Espectro EDS adquirido sobre los depósitos grises incluidos en la estratigrafía de la tola J7. Indica la presencia de fosfato $(\mathrm{P})$ asociado a calcio (Ca) correspondiendo a hidroxilapatita (Wright V., C2RMF, 2009) 


\section{INTERPRETACIONES}

\section{1. La tola J8}

Los análisis permitieron caracterizar algunos de los materiales empleados para construir la Estructura 1 (fig. 6): adobes compuestos de cuarzo, feldespatos e ilita, un enlucido grueso aplicado sobre la estructura de la misma composición que los adobes pero cocido, y un enlucido más fino y blanco aplicado en superficie compuesto de cuarzo, feldespatos, ilita, montmorillonita y carbonato de calcio, con una cola proteínica añadida. El uso de un enlucido posiblemente decorativo aplicado sobre la capa de tierra ya cocida parece demostrar que el enlucido grueso se coció intencionalmente. Quemar pisos de ocupación para endurecer su superficie es una práctica ya identificada en varios sitios ecuatorianos (Echeverría Almeida, 1996), y también en el sitio de Japoto mismo, en la tola J5 (Bouchard et al., 2006: 255). Finalmente, se han podido poner en evidencia algunos marcadores geológicos, indicios útiles en el futuro para trabajar sobre hipótesis acerca de la procedencia geológica de los materiales de construcción.

\begin{tabular}{|c|c|c|c|}
\hline Tipo de material & Adobes & $\begin{array}{l}\text { Enlucido grueso } \\
\text { quemado }\end{array}$ & Enlucido fino blanco \\
\hline Composicion & $\begin{array}{l}\text { Cuarzo } \\
\text { + Feldespatos } \\
\text { + Ilita }\end{array}$ & $\begin{array}{l}\text { Cuarzo } \\
\text { + Feldespatos } \\
\text { + Ilita }\end{array}$ & $\begin{array}{l}\text { Cuarzo } \\
\text { + Feldespatos } \\
\text { + ilita y montmorillonita } \\
\text { + Carbonato de Calcio } \\
\text { + cola proténica? }\end{array}$ \\
\hline
\end{tabular}

\section{2. La tola J7}

Se han podido desechar hipótesis relativas a la posible importancia de las propiedades acido básicas del suelo en el uso funerario de la tola y revelar la presencia de fosfatos de calcio que corresponden muy posiblemente a polvo de hueso. Queda ahora por determinar el carácter fortuito o intencional de estos depósitos.

\section{CONCLUSIONES}

Gracias a este estudio ha sido posible constatar que la arqueometría ofrece informaciones útiles para la resolución de problemas arqueológicos. Con 
respecto a la tola J8 se han obtenido datos sobre las técnicas y los materiales de construcción. En el caso de la tola J7, aunque los resultados sean preliminares, se han contribuido al entendimiento de las costumbres funerarias practicadas en Japoto.

Se demuestra por ende el interés de la arqueometría como una técnica de trabajo complementaria a la arqueología, confirmándose la importancia de proseguir los análisis comenzados.

\section{Referencias citadas}

BOUCHARD, J.-F., FUENTES, F. \& LÓPEZ, T., 2006 - Aldeas y pueblos prehispánicos en la costa de Manabí: Chirije y Japoto. Bulletin de l'Institut Français d'Études Andines, 35 (3): 243-256.

DELABARDE, T., 2006 - Una secuencia de patrones funerarios manteños en la provincia de Manabí: primeros resultados de la tola J7, sitio de Japoto (Ecuador). Bulletin de I'Institut Français d'Études Andines, 35 (3): 313-320.

EBERHART, J.-P., 1997 - Analyse structurale et chimique des matériaux, 614 pp.; París: Éditions Dunod.

ECHEVERRÍA ALMEIDA, J., 1996 - L'organisation des confédérations, Période d'Intégration (550-1530 apr. J.-C.). Les derniers Incas - Civilisations précolombiennes en Équateur; París: Éditions Faton. Les Dossiers d'Archéologie, n. 214.

GUINEA, M., 2006 - El uso de tierras comestibles por los pueblos costeros del Periodo de Integración en los Andes septentrionales. Bulletin de l'Institut Français d'Études Andines, 35 (3): 321-334.

PLATEL, N., 2006 - Rapport d'analyses, CRP2A - Université Michel de Montaigne Bordeaux 3. Non publié.

POMIES, M.-P., 1997 - Pigments rouges préhistoriques : goethite chauffée ou hématite nanocristalline ?, 248 pp.; París: Université de Paris 06. Travaux Universitaires - Tesis de doctoradot bajo la dirección de Michel Menu.

WRIGHT, V., 2008 - Étude de la polychromie des reliefs sur terre crue de la Huaca de la Luna Trujillo, Pérou, 285 pp.; Oxford: British Archaeological Reports (BAR S1808), Paris Monographs in American Archaeology 21 - Archaeopress. 